\title{
Strain effects on the magnetic order of epitaxial FeRh thin films
}

\author{
H. Kumar ${ }^{\mathrm{a}}$ and D. R. Cornejo \\ Laboratório de Materiais Magnéticos, \\ Departamento de Física dos Materiais e Mecânica, \\ Universidade de São Paulo, Brasil. \\ Sergio L. Morelhao \\ Instituto de Física Aplicada, Universidade de São Paulo, Brasil. \\ G. Alejandro and N. R. Álvarez \\ Centro Atómico Bariloche (CNEA) and Conicet, \\ 8400 Bariloche, Río Negro, Argentina. \\ I. M. Montellano \\ Instituto Balseiro (U. N. Cuyo), 8400 Bariloche, Río Negro, Argentina
}

\author{
A. Butera ${ }^{b}$ \\ Centro Atómico Bariloche (CNEA), \\ Instituto Balseiro (U. N. Cuyo), and Conicet, \\ 8400 Bariloche, Río Negro, Argentina.
}

(Dated: December 11, 2017) 


\begin{abstract}
In this work we report experimental results obtained on a set of $\sim 90 \mathrm{~nm}$ thick FeRh epitaxial films deposited on $\mathrm{MgO}(100), \mathrm{MgO}(111)$ and $\mathrm{Al}_{2} \mathrm{O}_{3}$ (0001) single crystal substrates. The magnetic characterization was achieved by measuring magnetization curves and ferromagnetic resonance as a function of temperature and orientation of the films with respect to the applied magnetic field. We discuss our results by comparing the characteristics of the antiferromagnetic-ferromagnetic transition among FeRh films of the same thickness but exposed to different post-growth annealings, and deposited on substrates of different crystalline orientation. We have found that there is a strong correlation between the strain present in the films and their magnetic behavior, observing that a change in the in-plane stress from compressive to tensile tends to shift the magnetic transition by more than $60 \mathrm{~K}$. The interplay between magnetic and elastic properties was further analyzed by ferromagnetic resonance and we have found that the magnetoelastic component of the anisotropy varies from out-of-plane to in-plane, depending on the substrate. These findings could be of great importance if a precise tuning of the magnetic transition temperature or the magnetic anisotropy is needed for a specific application.
\end{abstract}

PACS numbers: $75.70 . \mathrm{Ak}, 76.50 .+\mathrm{g}, 75.30 . \mathrm{Gw}, 75.50 . \mathrm{Bb}$

Keywords: FeRh, thin magnetic films, magnetic transition, ferromagnetic resonance 


\section{INTRODUCTION}

It is well known that bulk FeRh undergoes a very unusual transition from an antiferromagnetic $(\mathrm{AF}) \alpha^{\prime \prime}$ to a ferromagnetic (FM) $\alpha^{\prime}$ phase upon heating from room temperature (RT) to above $T_{\mathrm{AF}-\mathrm{FM}} \approx 300-370 \mathrm{~K}$ without developing structural changes. ${ }^{1-3}$ In the $\alpha "$ phase, Fe spins are ordered AF in the [001] direction, with a net magnetic moment of 3.3 $\mu_{B}$, while Rh ions display no magnetic moment. In the $\alpha^{\prime}$ phase, all the spins are aligned ferromagnetically, also in the [001] direction, with a magnetic moment of $3.2 \mu_{B}$ contributing from the Fe and $0.9 \mu_{B}$ from the Rh atoms.

The crystal structure of the $\mathrm{Fe}_{50} \mathrm{Rh}_{50}$ alloy is $\mathrm{CsCl}$ type, and the AF-FM transition is of first order with a thermal hysteresis of the order of $10 \mathrm{~K}$ in bulk materials. In Fig. 1 we reproduce schematically the bulk phase diagram reported in Ref. 3, focused on the magnetic behavior around the $50 \%$ composition region. Note that the AF-FM transition temperature tends to increase by more than $50 \mathrm{~K}$ when the Rh concentration changes from $50 \%$ to $55 \%$. Fe-rich alloys display a bcc $\alpha$ phase that converts into the paramagnetic (PM) fcc $\gamma$ phase at high temperatures. The $\alpha \leftrightarrow \gamma$ transition is of martensitic type. ${ }^{4}$ According to Ref. 2 the $\gamma$ phase can be retained at room temperature by rapid quenching from the $\gamma$ field for alloys between $25 \%$ and $40 \%$ Rh. In thin films, the phase diagram and their boundaries are not clearly established yet, so the presence of small amounts of secondary phases, like the bcc $\alpha(\mathrm{FM})$ or fcc $\gamma(\mathrm{PM})$, is often observed in nearly equiatomic FeRh films.

Although FeRh is not the only compound that develops this unusual AF to FM transition, ${ }^{5}$ it is indeed unique in the sense that $T_{\mathrm{AF}-\mathrm{FM}}$ is significantly above room temperature. Beyond the basic interest in the underlying physics, this feature makes FeRh a very interesting material for technological applications, such as thermally assisted magnetic recording ${ }^{6}$ or resistive memories. ${ }^{7}$ Although the bulk binary system (Fe, Rh) has been extensively studied since the 1940's and rather complete phase diagrams were obtained, work in FeRh thin films has only started relatively recently because of the potential applications in magnetic storage ${ }^{6,8-14}$ It was already shown that substitutional doping, ${ }^{15}$ magnetic field,${ }^{9}$ strain, ${ }^{16}$ film thickness, ${ }^{11}$ etc. can be used to modify and tune the magnetic behavior. To complement those studies, we report in this article the interplay between magnetism and substrate induced strain effects in FeRh thin films deposited on different single crystal wafers. 


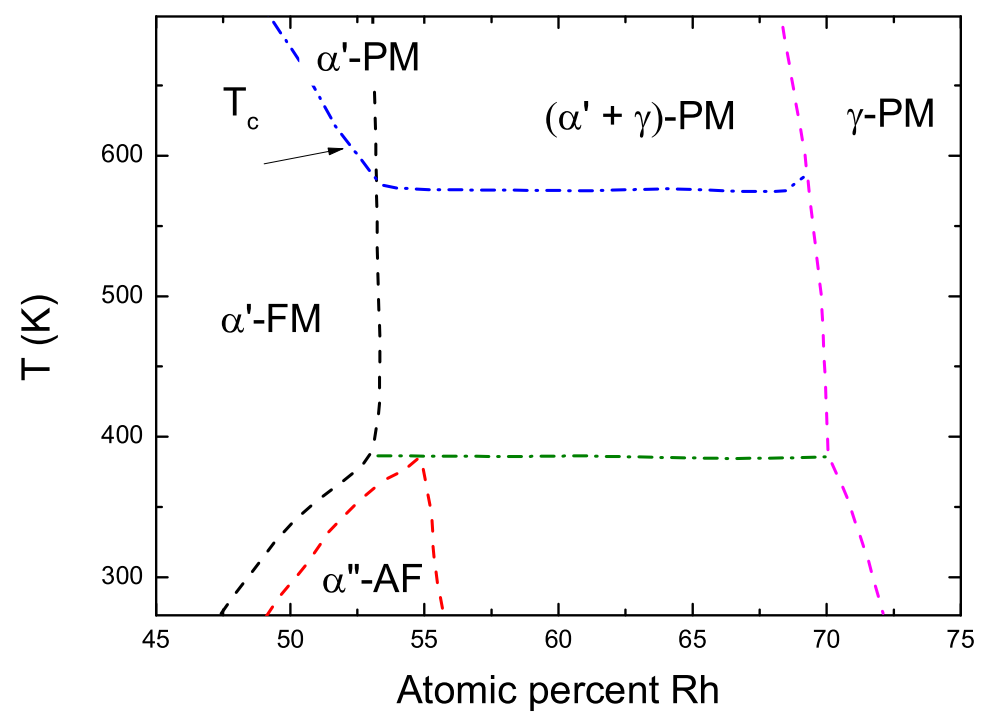

FIG. 1: Phase diagram of the binary ( $\mathrm{Fe}, \mathrm{Rh})$ bulk alloy around the equiatomic composition, adapted from Ref. 3.

\section{EXPERIMENTAL PROCEDURE}

FeRh thin films with nominally equiatomic composition were deposited at 5 mTorr $\mathrm{Ar}$ pressure onto $\mathrm{MgO}(100), \mathrm{MgO}(111)$ and $\mathrm{Al}_{2} \mathrm{O}_{3}$ (0001) $\left(c-\mathrm{Al}_{2} \mathrm{O}_{3}\right)$ single crystal substrates by dc magnetron sputtering from separate Fe and Rh targets (99.99\% purity) in an AJA 2000 sputtering system. The deposition process to obtain films with good epitaxial grow was optimized at $T=525^{\circ} \mathrm{C}$, a rate of $\sim 0.03 \mathrm{~nm} / \mathrm{s}$, and a base pressure of $1.2 \times 10^{-6}$ Torr. The nominal thickness of the films was chosen to approximately $90 \mathrm{~nm}$ and was afterwards determined by Rutherford Back Scattering Spectrometry (RBS). The crystal structure of the FeRh thin films was probed with x-ray diffraction experiments (XRD), that were conducted in the Rigaku x-ray diffraction system with the 3-circle Huber diffractometer of the XRD2 beamline of Brazil Synchrotron facility (LNLS, Campinas), using a wavelength $\lambda=0.154056$ $\mathrm{nm}$. To study the change in sample volume while traversing through the magnetostructural transition, additional diffraction studies of a symmetric FeRh reflection were performed in selected samples as a function of temperature in the range $300 \mathrm{~K} \leq T \leq 558 \mathrm{~K}$.

The magnetic characterization of the samples was accomplished by performing dc magnetization $(M)$ and ferromagnetic resonance (FMR) experiments. The magnetization vs. 
temperature data were collected with the magnetic field $(H)$ applied parallel to the film plane. FMR spectra were acquired using a commercial Bruker ESP300 spectrometer operating at a microwave frequency of $9.4 \mathrm{GHz}$ (X band). The samples were placed in the center of a rectangular resonant cavity, where the derivative of the absorbed power was measured using standard field modulation and lock-in detection techniques with amplitudes in the range 5-20 Oe. The samples could be rotated inside the resonator in order to collect the spectra for different orientations of the films, and the temperature dependent experiments were performed in the range $300-480 \mathrm{~K}$.

\section{EXPERIMENTAL RESULTS}

\section{A. Film growth and epitaxy}

The composition and thickness of each sample were determined by using RBS. For the films used in this study we have determined a 55\% at. Rh and a thickness of $92 \mathrm{~nm}$. The rest of the relevant parameters are summarized in Table I. Most of the samples were post-annealed at $\mathrm{T} \sim 700{ }^{\circ} \mathrm{C}$ for four hours in vacuum to promote the atomic order of Fe and Rh species. The quality of the films was tested by x-ray diffraction measurements at room temperature. We have found that in all cases the diffraction patterns show a majority $\alpha$-bcc phase with traces of the $\gamma$-fcc structure in some films, which could not be completely removed even after annealing. XRD results also indicate (see Fig. 2) that all films grew with a strong texture that depends on the type of substrate. It is clear from the figure that annealed films deposited on $\mathrm{MgO}(100), \mathrm{MgO}(111)$ and $c-\mathrm{Al}_{2} \mathrm{O}_{3}$ substrates develop strong [001], [011] and [111] textures, respectively. As an exception, the as-grown film on $c-\mathrm{Al}_{2} \mathrm{O}_{3}$ (not shown) grows with a predominant [011] texture which turns to a [111] (superstructure) preferred orientation after annealing, indicating a higher degree of atomic order. The room temperature out-of-plane lattice parameter $(c)$ can be estimated from the interplanar distances of the (200), (220) and (111) Bragg reflections of FeRh films deposited on $\mathrm{MgO}(100), \mathrm{MgO}(111)$ and $c-\mathrm{Al}_{2} \mathrm{O}_{3}$ substrates. We obtained $c=0.3021(3)$ $\mathrm{nm}, c=0.2993(3) \mathrm{nm}$ and $c=0.2980(3) \mathrm{nm}$, respectively. We can compare these values with the measured relaxed lattice parameter of $\mathrm{Fe}_{45} \mathrm{Rh}_{55}$ epitaxial films, $a_{0}=0.3000(2)$ $\mathrm{nm}$, obtained from the out of plane and in-plane measurements of the lattice parameter 
TABLE I: Name of the different samples used in this study with their relevant parameters. In all cases the Rh concentration is $55 \%$ atomic and the film thickness is $92 \mathrm{~nm}$. We indicate in bold characters the dominant crystalline texture

\begin{tabular}{|c|c|c|c|c|}
\hline Sample & Substrate & Post-annealing & Orientation of the film & $\varepsilon_{z}$ \\
\hline $7 \mathrm{M} 100$ & $\mathrm{MgO}(100)$ & $700{ }^{\circ} \mathrm{C}$ & {$[\mathbf{1 0 0}] \mathbf{b c c}$} & $6.9 \times 10^{-3}$ \\
\hline $7 \mathrm{M} 111$ & $\mathrm{MgO}(111)$ & $700{ }^{\circ} \mathrm{C}$ & {$[\mathbf{1 1 0}] \mathbf{b c c}+[111]$ fcc } & $-2.4 \times 10^{-3}$ \\
\hline $7 \mathrm{~A} 01$ & $\mathrm{Al}_{2} \mathrm{O}_{3}(0001)$ & $700{ }^{\circ} \mathrm{C}$ & {$[\mathbf{1 1 1}] \mathbf{b c c}+[200]$ fcc } & $-6.9 \times 10^{-3}$ \\
\hline $\mathrm{A} 01$ & $\mathrm{Al}_{2} \mathrm{O}_{3}(0001)$ & No & {$[\mathbf{1 1 0}] \mathbf{b c c}+[111]$ fcc } & - \\
\hline
\end{tabular}

of $\mathrm{Fe}_{45} \mathrm{Rh}_{55}$ on $\mathrm{MgO}(100)$. This value is coincident, within error, with that reported in polycrystalline films ( $\alpha$ " phase) deposited on Si substrates, $a_{0}=0.2994(7) \mathrm{nm} .{ }^{14}$

In the case of the sample 7M100 the FeRh(100) plane tends to grow rotated by $45^{\circ}$ with respect to the $\mathrm{MgO}(100)$ substrate. In this situation the lattice mismatch between $\mathrm{Fe}_{45} \mathrm{Rh}_{55}(100)$ and $\mathrm{MgO}(100)$ substrate $\left(a_{\mathrm{MgO}}=0.4198=\sqrt{2} 0.2978 \mathrm{~nm}\right)$ produces a tetragonal distortion of the FeRh cubic unit cell which, as expected, is expanded along the out-of-plane [001] direction and compressed along in-plane [100] and [010] directions. From these values we obtained $\varepsilon_{z}=\left(c-a_{0}\right) / a_{0}=6.9 \times 10^{-3}$, and using the reported ${ }^{17}$ Poisson's ratio, $v \sim 0.32$, we can estimate the in-plane strain $\varepsilon_{x}$ in the case of uniform biaxial stress, $\varepsilon_{x}=(\nu-1) /(2 \nu) \varepsilon_{z} \sim-7.3 \times 10^{-3}$. With this value of $\varepsilon_{x}$ and the relaxed lattice parameter $a_{0}$ we estimated the in-plane lattice parameter $a=0.2978(3) \mathrm{nm}$, which is similar to the interatomic $\mathrm{MgO}$ spacing, indicating that the FeRh film is affected by the substrate. Note that although the films have been fabricated above the AF-FM transition and that FeRh undergoes a relatively large decrease in volume at the magnetic transition when cooling down (as observed in our own measurements and also reported in Refs. 18 and 19), the FeRh lattice still matches that of the $\mathrm{MgO}$ substrate.

The quality of the out-plane data suggests that the films are epitaxially oriented. This was confirmed by performing an asymmetric phi-scan on the $\mathrm{Fe}_{45} \mathrm{Rh}_{55}$ film and comparing it to that corresponding to the substrate. The in-plane epitaxial relation of the $\mathrm{Fe}_{45} \mathrm{Rh}_{55}(100)$ film on $\mathrm{MgO}(100)$ was explored by first aligning to the symmetric $\mathrm{MgO}(100)$ and asymmetric $\mathrm{MgO}(420)$, and then aligning to symmetric $\mathrm{Fe}_{45} \mathrm{Rh}_{55}(100)$ and asymmetric $\mathrm{Fe}_{45} \mathrm{Rh}_{55}(210)$ at room temperature. 


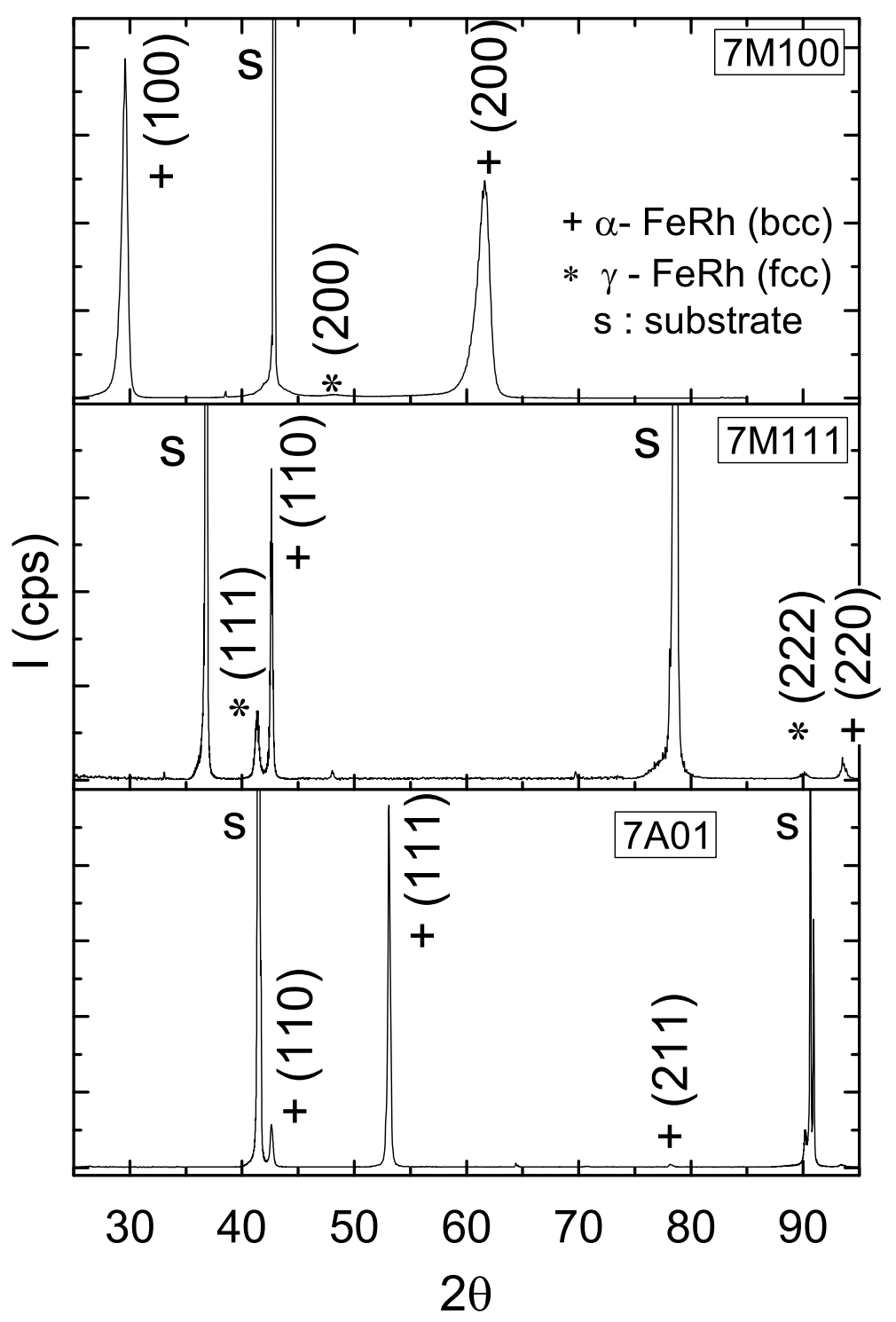

FIG. 2: X-ray diffraction patterns of $\mathrm{Fe}_{45} \mathrm{Rh}_{55}$ films deposited on $\mathrm{MgO}(100), \mathrm{MgO}(111)$ and $\mathrm{Al}_{2} \mathrm{O}_{3}(0001)$ wafers. Diffractions peaks from the film and from the substrate are indicated on the patterns.

The phi-scan diffractograms are shown in Fig. 3 and indicate that both the $\operatorname{MgO}(100)$ substrate and the $\mathrm{Fe}_{45} \mathrm{Rh}_{55}(100)$ oriented film have four-fold symmetry. As can be seen in the figure the matching between substrate and film is better fulfilled if the [100] direction of the film is rotated by $45^{\circ}$ with respect to the [100] direction of the substrate. 


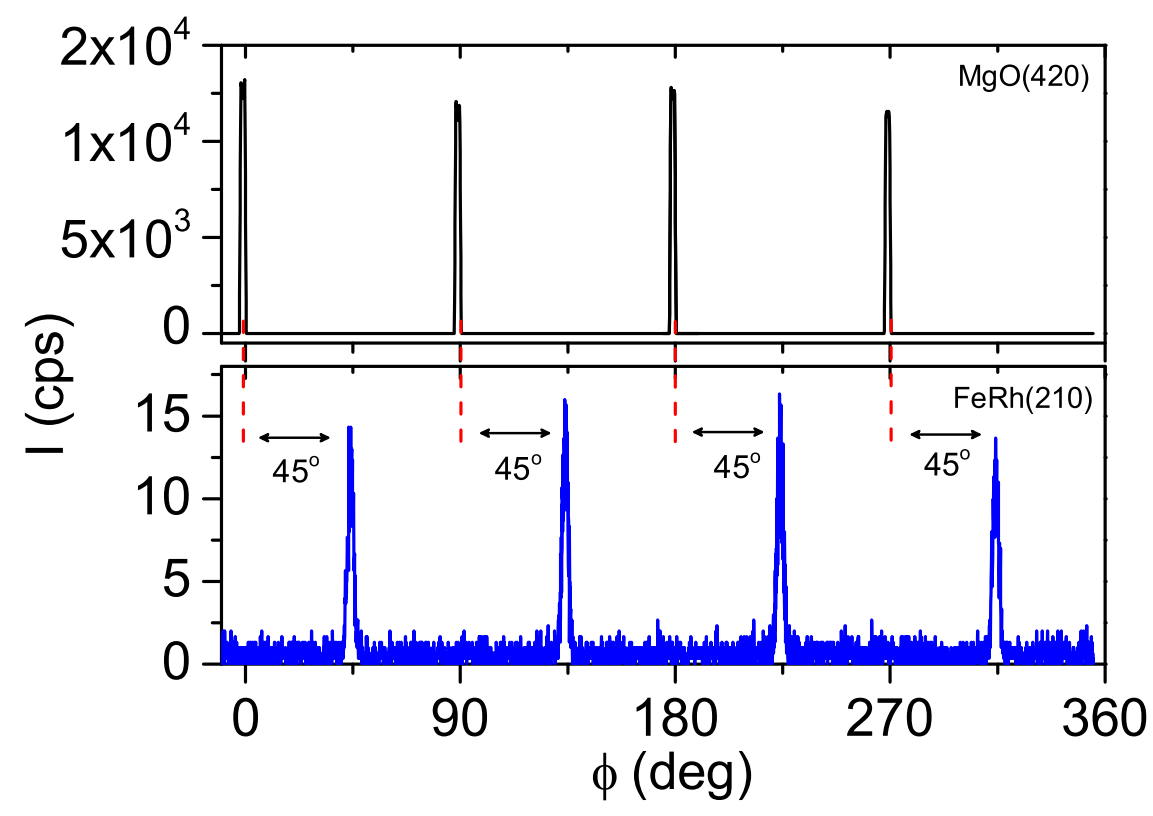

FIG. 3: Phi-scan diffractograms of the $7 \mathrm{M} 100$ sample showing the $45^{\circ}$ rotation of the FeRh cubic cell with respect to the $\mathrm{MgO}(100)$ substrate. The radial angle was set to $\chi=26.6^{\circ}$.

In the case of $\mathrm{MgO}(111)$ substrates, the films tend to grow with a strong [011] texture of the bcc phase, and a minor [111] textured fcc-phase, which could not be removed even after annealing at $700{ }^{\circ} \mathrm{C}$. Two different epitaxies are often found when bcc compounds are deposited on fcc (111) surfaces. In both situations the matching plane is the bcc $(110),{ }^{20}$ but in one case the in-plane orientation relationship is [110]bcc // [112]fcc (NishiyamaWasserman), and in the other [111]bcc // [110]fcc (Kurdjumov-Sachs). From our XRD patterns it was not possible to distinguish which of the two variants was predominant, but we have found that even though the lattice mismatch between both lattices is relatively large, the interplanar (110) distance perpendicular to the film plane $\left(d_{110}=0.2117(2) \mathrm{nm}\right)$ is slightly smaller than that expected for a relaxed FeRh lattice $\left(d 0_{110}=0.2121 \mathrm{~nm}\right)$, giving a strain $\varepsilon_{z}=-2.4 \times 10^{-3}$.

As we have already mentioned [110] and [111] textures were observed in as-deposited and annealed films on $c-\mathrm{Al}_{2} \mathrm{O}_{3}$. In this aspect Yuasa et al. ${ }^{21}$ reported that the epitaxial nature of $(\mathrm{Fe}, \mathrm{Rh})_{95} \mathrm{Ir}_{5}$ films deposited on $\mathrm{Al}_{2} \mathrm{O}_{3}(0001)$ substrates depends upon the sputtering rate, in such a way that in the range $0.02 \mathrm{~nm} / \mathrm{s}-0.06 \mathrm{~nm} / \mathrm{s}$ both (110) or (111) oriented films can be obtained. In our case annealed FeRh films deposited on $c-\mathrm{Al}_{2} \mathrm{O}_{3}$ substrates develop a 
strong [111] out of plane texture. The epitaxial growth was further analyzed using phi-scans. We have found an in-plane epitaxy with the relationship $\mathrm{Fe}_{45} \mathrm{Rh}_{55}\left[\begin{array}{lll}-1 & -1 & 2\end{array}\right] / / \mathrm{Al}_{2} \mathrm{O}_{3}\left[\begin{array}{lll}0 & -1\end{array}\right.$ 0]. As the in-plane cell size is larger than that corresponding to the film by approximately $10 \%$, an in-plane tensile strain is expected for FeRh on $c-\mathrm{Al}_{2} \mathrm{O}_{3}$. This, in turn produces a compressive strain in the out-of-plane direction. From the diffractograms we have deduced the interplanar distances $d_{111}=0.1720(2) \mathrm{nm}$ and $d 0_{111}=0.1732(2)$ which can be used to estimate the out-of-plane and in-plane strains, $\varepsilon_{z}=-6.9 \times 10^{-3}$ and $\varepsilon_{x}=7.2 \times 10^{-3}$.

Although the composition of our films is different from that reported in Ref. 9 a similar trend for the strain of $\mathrm{FeRh}$ on $\mathrm{MgO}(100)$ and $c-\mathrm{Al}_{2} \mathrm{O}_{3}$ was found. Note that by using different substrates the in-plane strain can be changed from compressive $(\mathrm{MgO}(100))$ to tensile $\left(c-\mathrm{Al}_{2} \mathrm{O}_{3}\right)$, which is expected to reflect in the magnetic properties of the films. For example, the tensile stress of FeRh films on the sapphire substrate could be expected to decrease the AF-FM transition temperatures because the larger cell size induces the stabilization of the ferromagnetic phase at lower temperatures. ${ }^{16}$

\section{B. Magnetization}

In Fig. 4 we plot typical magnetization vs. temperature cycles, obtained from samples 7M100 (top), 7M111 (center) and 7A01-A01 (bottom) while cooling and heating them inside a Faraday balance magnetometer with an applied field $H=1200$ Oe parallel to the film plane.

At room temperature the magnetization is very close to zero for samples 7M100 and 7M111 (annealed), which is consistent with the $\alpha$ "-AF state. Upon heating, the magnetization gradually increases according to the growing fraction of the $\alpha^{\prime}$-FM phase. The maximum magnetization value for these films reaches $M_{s}^{\max } \sim 1025 \mathrm{emu} / \mathrm{cm}^{3}$ at $T=392$ $\mathrm{K}$ in the cooling branch of the 7M111 film. This value of $M_{s}$ is within the broad range of reported data ${ }^{9-11,13,22,23}$ for different FeRh films with $M_{s}^{\max }=900-1200 \mathrm{emu} / \mathrm{cm}^{3}$. For temperatures above $420 \mathrm{~K}$ the AF-FM transition seems to be complete for all samples, but a strong dependence with the substrate was found. Upon cooling we observe a hysteretic behavior, which confirms the first order character of the phase transition.

We have defined the transition temperatures $T_{\mathrm{AF}-\mathrm{FM}}^{h}$ (heating) and $T_{\mathrm{AF}-\mathrm{FM}}^{c}$ (cooling) as the points where the magnetization reaches $90 \%$ of its maximum, and the corresponding 


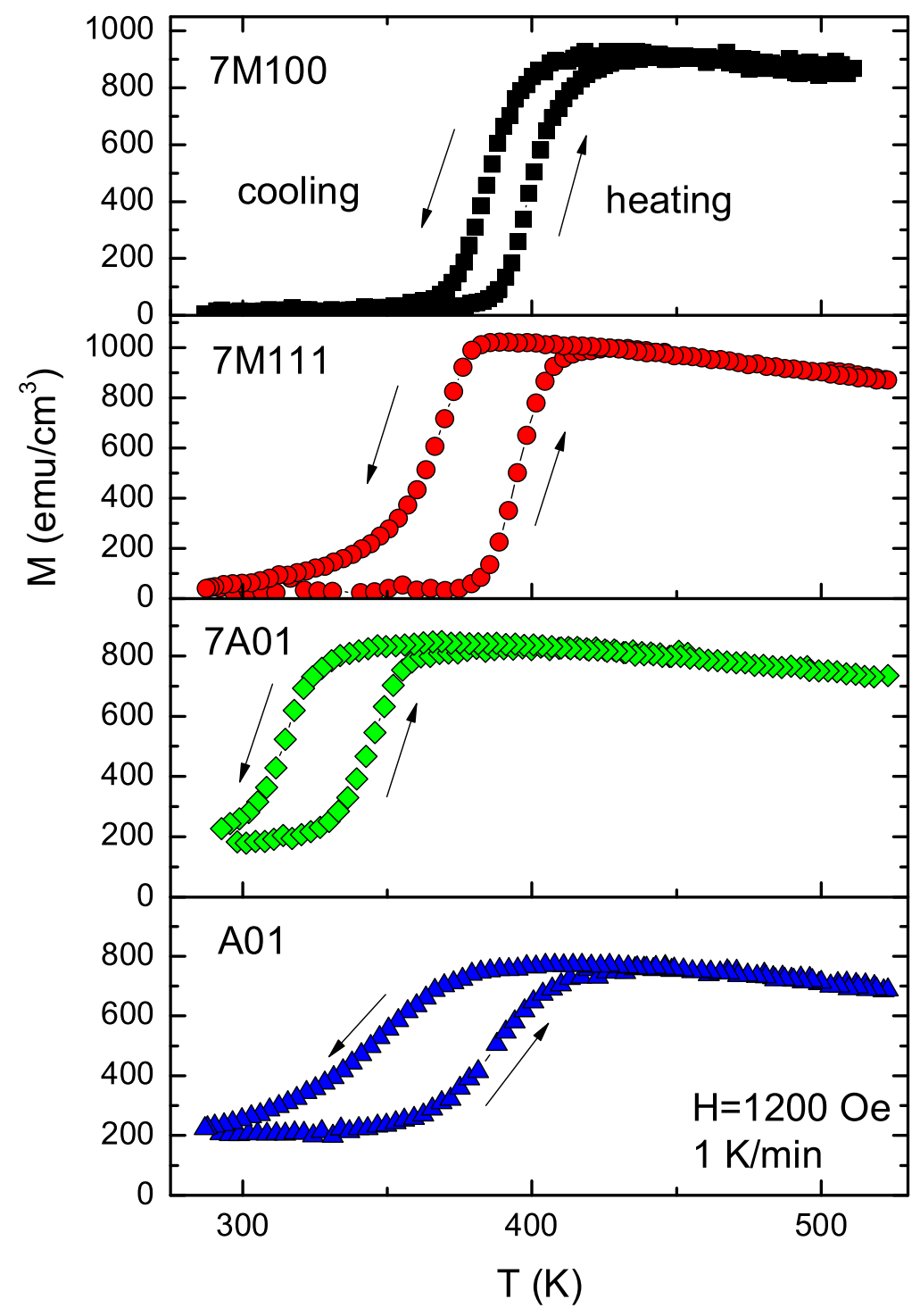

FIG. 4: Magnetization vs. temperature cycles performed on samples 7M100, 7M111, 7A01 and A01. The static magnetic field $H=1200$ Oe was applied parallel to film plane at a heating/cooling rate of $1 \mathrm{~K} / \mathrm{min}$.

difference $\Delta T=T_{\mathrm{AF}-\mathrm{FM}}^{h}-T_{\mathrm{AF}-\mathrm{FM}}^{c}$. In Table II we summarize the results for the $55 \% \mathrm{Rh}$ samples: 7M100, 7M111, 7A01, and A01. In the case of annealed films the AF-FM transition of the heating branch decreases from $T_{\mathrm{AF}-\mathrm{FM}}^{h}=418 \mathrm{~K}$ for $7 \mathrm{M} 100$ to $T_{\mathrm{AF}-\mathrm{FM}}^{h}=355 \mathrm{~K}$ for 7A01. As already mentioned when the crystallographic results were discussed, this behavior 
TABLE II: Transition temperature $T_{\mathrm{AF}-\mathrm{FM}}$ (heating and cooling the films) and width of the thermal hysteresis estimated from dc magnetization and FMR experiments (all in K). The error in the determination of the temperature is indicated in parentheses.

\begin{tabular}{|c|c|c|c|}
\hline Sample & $\begin{array}{c}\mathrm{T}_{\text {AF-FM }}(\text { heating) } \\
\text { DC/FMR }\end{array}$ & $\begin{array}{c}\mathrm{T}_{\text {AF-FM }} \text { (cooling) } \\
\text { DC/FMR }\end{array}$ & $\begin{array}{c}\Delta \mathrm{T}(\mathrm{K}) \\
\text { DC/FMR }\end{array}$ \\
\hline 7M100 & $418 / 410$ & $398 / 400$ & $20 / 10(5)$ \\
\hline 7M111 & $406 / 398$ & $376 / 375$ & $30 / 23(5)$ \\
\hline 7A01 & $355 / 360$ & $327 / 325$ & $28 / 25(5)$ \\
\hline A01 & $408 / 425$ & $368 / 400$ & $40 / 25(5)$ \\
\hline
\end{tabular}

is consistent with the systematic change that occurs in the in-plane strain, from compressive to tensile, when $\mathrm{MgO}(100), \mathrm{MgO}(111)$ and $c-\mathrm{Al}_{2} \mathrm{O}_{3}$ are used as substrates. The transition temperature $T_{\mathrm{AF}-\mathrm{FM}}^{h}=418 \mathrm{~K}$ for $7 \mathrm{M} 100$ is considerably larger than the values reported in Refs. 9-11,23, which average $T_{\mathrm{AF}-\mathrm{FM}}^{h}=400 \mathrm{~K}$. However, all those films correspond to the composition $\mathrm{Fe}_{49} \mathrm{Rh}_{51}$, while our samples have $55 \%$ Rh, which was already shown ${ }^{2}$ in Fig. 1 to shift the transition to higher temperatures. Apart from composition, differences may also arise in the strong dependence of the transition temperature on the applied magnetic field, film thickness, ${ }^{24}$ and the magnetic and thermal history. ${ }^{9}$ For the sample 7A01 we obtained $T_{\mathrm{AF}-\mathrm{FM}}^{h}=355 \mathrm{~K}$ which is almost the same as the value reported in Ref. $9\left(T_{\mathrm{AF}-\mathrm{FM}}^{h} \sim 360\right.$ $\mathrm{K})$. The width of the temperature hysteresis $(\Delta T)$ is smaller in $7 \mathrm{M} 100(\Delta T \sim 20 \mathrm{~K})$ than in 7M111 and 7A01 $(\Delta T \sim 30 \mathrm{~K})$. This could be due to the presence of a relatively larger fraction of the $\gamma$-paramagnetic phase in the last two samples, ${ }^{11}$ as seen in the diffraction data. In the case of the non annealed A01 sample (and also in 7A01), the magnetization takes an appreciable value $M \sim 200 \mathrm{emu} / \mathrm{cm}^{3}$ at $\mathrm{RT}$, reaching $M_{s}^{\max } \sim 770 \mathrm{emu} / \mathrm{cm}^{3}$ and $\Delta T=40 \mathrm{~K}$. All these features are consistent with the presence of two different bcc textures and the fcc $\gamma$-phase, which tend to stabilize the FM phase in the non-annealed sample.

\section{Ferromagnetic resonance (FMR)}

To follow the evolution of the magnetic phases and determine the presence of anisotropies, we recorded FMR spectra at different temperatures, both heating and cooling from RT up to $\sim 480 \mathrm{~K}$ in each sample. In these experiments the static magnetic field was applied 
perpendicular to the film plane, as we found that the conditions for the detection of the resonant absorption of the incident microwaves were optimal for this orientation.

A standard FMR spectrum is the first derivative of the microwave power absorption of the sample with respect to the "static" magnetic field, $H$, which is slowly swept across a predefined field range. The field value at which the derivative is zero is defined as the resonance field $\left(H_{\text {res }}\right)$, and the distance between a derivative maximum and minimum is called the peak-to-peak linewidth $\left(\Delta H_{\mathrm{pp}}\right)$.

In Fig. 5 we plot a series of typical spectra recorded while decreasing the temperature from $455 \mathrm{~K}$ to $360 \mathrm{~K}$ for the sample $7 \mathrm{M} 111$. The qualitative features of the rest of the annealed samples are quite similar, although the characteristic temperatures are different. From $T \approx 380 \mathrm{~K}$ and above the FMR line is well defined and we can safely assume that the sample is completely in the $\alpha^{\prime}-\mathrm{FM}$ phase. We observe that when the temperature is decreased, the resonance field moves to higher values. At the same time, the linewidth increases and the intensity progressively diminishes until it virtually vanishes, and we can suppose that the sample is completely in the $\alpha$ "-AF phase. From the spectra measured for the different films we extracted the temperature behavior of the resonance field and the linewidth. In Fig. 6 we present these variables as a function of $T$ for the 7M100 sample. The temperature behavior of the FMR resonance parameters obtained for the 7M100 film are representative of the other annealed samples, so the following discussion applies for all of them.

The FMR cycles also present temperature hysteresis $(\Delta T)$ whose value depends on the substrate, as we can see from Table II. We can distinguish two different temperature regimes, which is more evident in $H_{\text {res }}$ vs. $T$ in Fig. 6(a): a "high- $T$ " regime where $H_{\text {res }}$ increases when the temperature is decreased until it reaches a maximum, and a "low- $T$ " regime where $H_{\text {res }}$ diminishes rapidly with decreasing temperature. From the $H_{\text {res }}$ vs. $T$ curves we obtained the temperature of the magnetic transition $\left(T_{\mathrm{AF}-\mathrm{FM}}\right)$, by determining the point at which $H_{\text {res }}$ vs. $T$ is maximum. When we cool the sample from the high temperature $\alpha$ '-FM region down to $\mathrm{RT}$, we approach gradually the temperature and field where the sample becomes entirely AF. Thus, the maximum of the $H_{\text {res }}$ vs. $T$ curve signals the temperature where the AF phase progressively begins to nucleate and the AF and FM phases start to coexist. In Table II we summarize the values of $T_{\mathrm{AF}-\mathrm{FM}}$ (heating and cooling) and the 


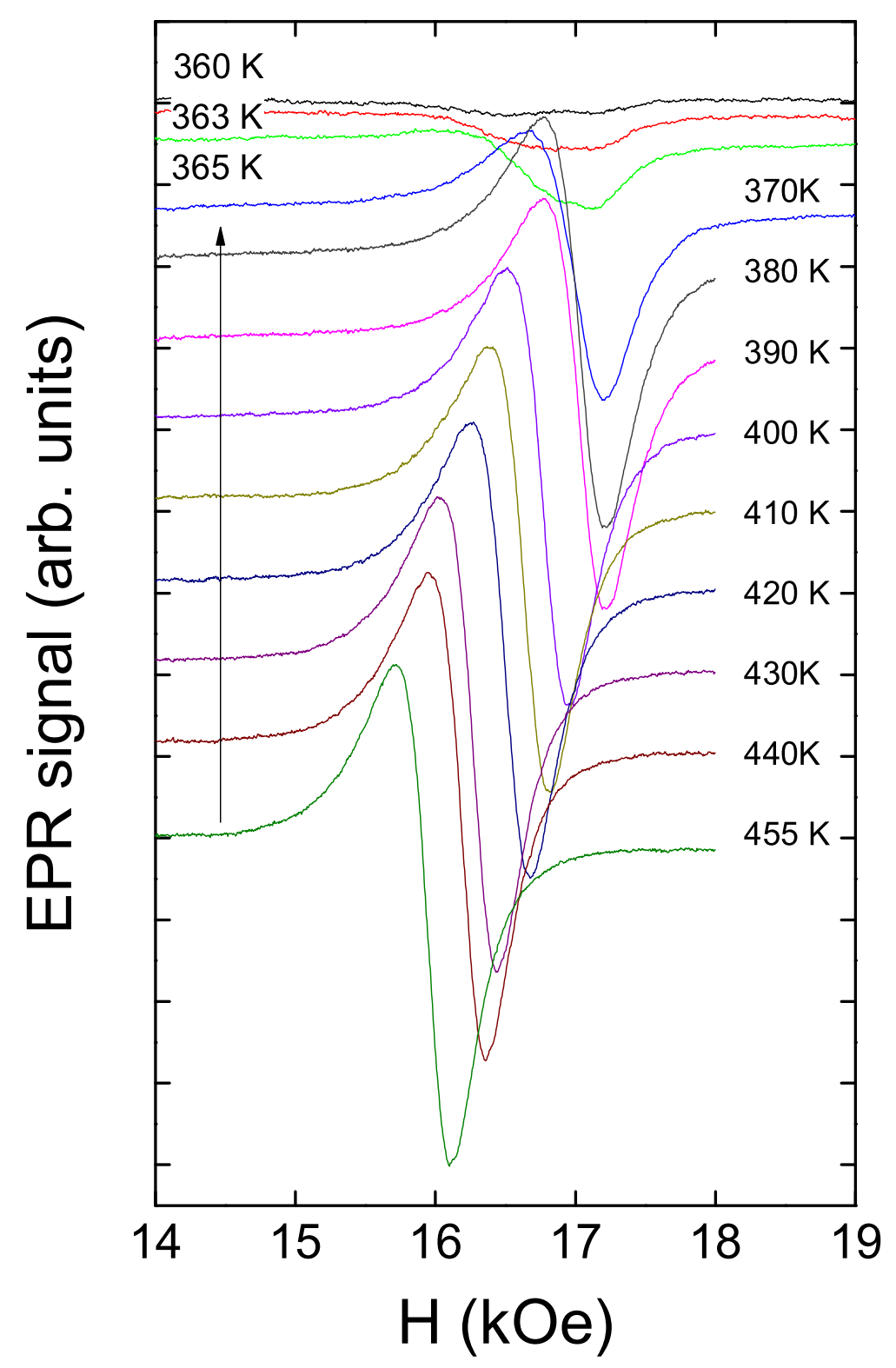

FIG. 5: FMR spectra of the annealed 7M111 sample recorded while cooling. The static magnetic field $H$ was set normal to the film plane. Spectra have been vertically shift for clarity. A similar behavior was observed for the other films.

width of the thermal hysteresis $(\Delta T)$ for the measured samples and compare these values with those obtained from dc magnetization experiments. A very good agreement is observed in the case of annealed films, even though the criterion for the determination of $T_{\mathrm{AF}-\mathrm{FM}}$ is not necessary equivalent in the two experimental techniques. These results also show that 


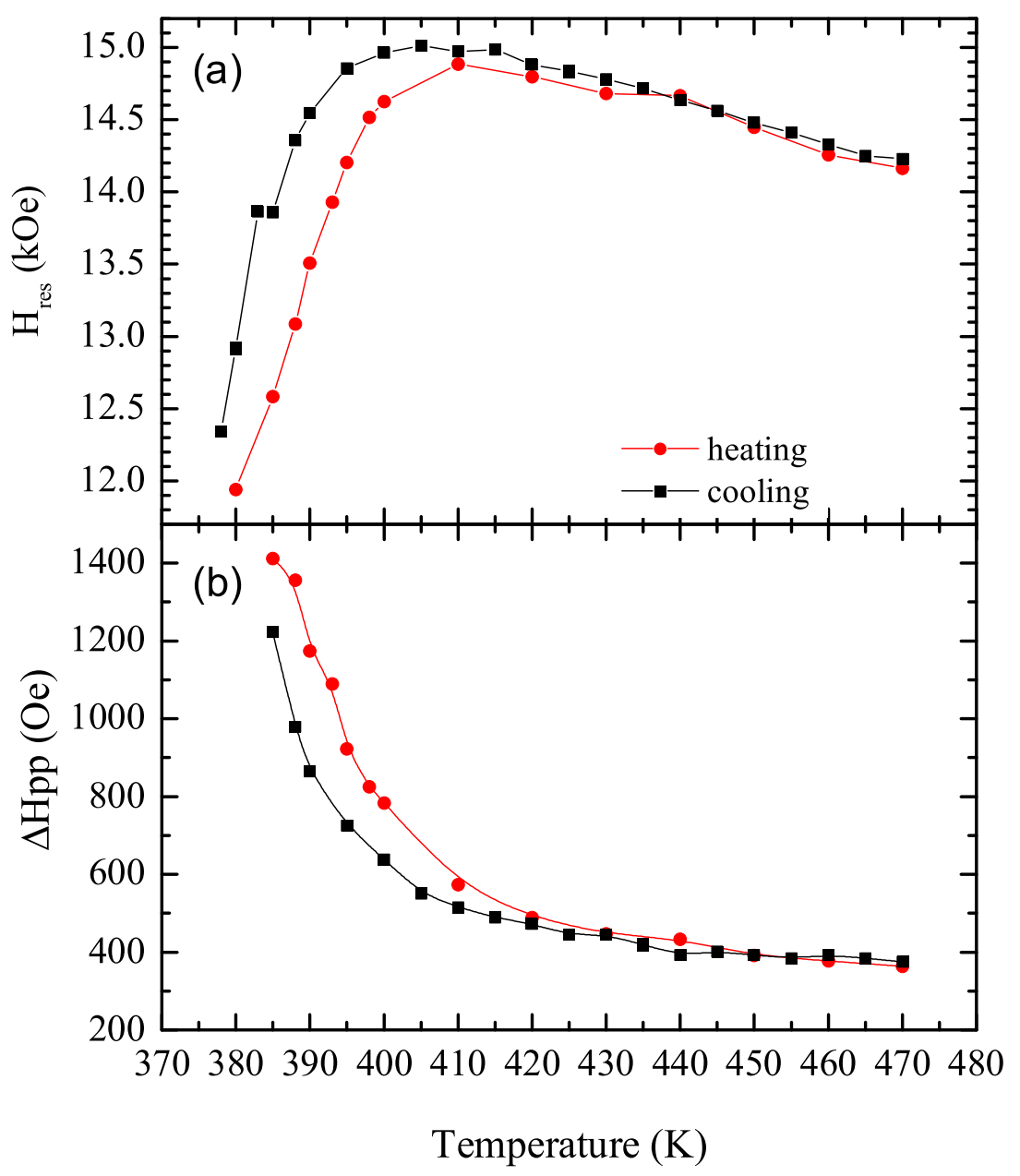

FIG. 6: $H_{\text {res }}\left(\right.$ a) and linewidth, $\Delta H_{\mathrm{pp}}$ (b), as a function of temperature for the annealed 7M100 sample. The experiments were done subsequently heating (circles) and cooling (squares).

$T_{\mathrm{AF}-\mathrm{FM}}$ is clearly substrate-dependent and reveal the influence of stress/strain effects due to the lattice mismatch between substrate and film. The highest and lowest $T_{\mathrm{AF}-\mathrm{FM}}$ were measured on the 7M100 and 7A01 films, respectively, which is fully consistent with the magnetization results.

Ferromagnetic resonance can also be used to obtain information about the magnetic anisotropies of the system. The anisotropy terms generally found in ferromagnetic films 
are in most cases of magnetocrystalline, magnetoelastic, or shape (dipolar) origin. The magnetocrystalline anisotropy is an intrinsic property of the material closely related to the spin orbit coupling, and usually reflects the symmetry of the crystal structure. The origin of the magnetoelastic anisotropy is usually extrinsic, and related to residual stresses introduced in the films during the growth process that induce a preferential axis (or plane) of easy magnetization. The shape anisotropy is a direct consequence of the dipolar interaction which produces a demagnetizing field, and in the case of a thin film provides an easy-plane of magnetization parallel to the film.

In order to study the magnetic anisotropies of our samples we performed FMR experiments varying the orientation of the external field, $H$, with respect to the film and collecting the resonance spectra at different angles. We performed the experiments using the out-ofplane (OOP) experimental setup where $H$ is rotated from the film plane to the normal of the film. In some cases we also acquired spectra rotating the magnetic field parallel to the film plane. From these FMR measurements it is possible to obtain information about the anisotropies present in the samples.

Experiments were recorded for all samples for $T>T_{\mathrm{AF}-\mathrm{FM}}$, choosing the temperature to ensure that the film was completely in the ferromagnetic phase. In Fig. 7 we show an OOP angular variation of the resonance field $H_{\text {res }}$ for the sample 7M100. A similar behavior was observed in the other films. The OOP angular variation of $H_{\text {res }}$ in Fig. 7 is typical of a thin film for which the easy plane of magnetization coincides with the film itself, as expected.

To quantify our results we used the Smit-Beljers model for a thin film ${ }^{25}$ with shape plus uniaxial anisotropy with the same symmetry, which leads to the Kittel expressions with an effective field $H_{\text {eff }}$ that can be estimated from the resonance field parallel $\left(H_{/ /}\right)$and perpendicular $\left(H_{\perp}\right)$ to the film plane. ${ }^{26,27}$

$$
\begin{aligned}
& \frac{\omega}{\gamma}=\sqrt{H_{/ /}\left(H_{/ /}+H_{\mathrm{eff}}\right)}, \\
& \frac{\omega}{\gamma}=H_{\perp}-H_{\mathrm{eff}},
\end{aligned}
$$

where $H_{\text {eff }}=4 \pi M_{s}-H_{A}^{\perp} .4 \pi M_{s}$ is the shape anisotropy of a thin film and $H_{A}^{\perp}$ accounts for additional contributions to the magnetic anisotropy normal to the film plane. $\omega=2 \pi f$ is the driving angular frequency and $\gamma=g \mu_{B} / \hbar$, is the gyromagnetic factor, with $g \sim 2.09$ for Fe and ferromagnetic iron alloys, ${ }^{27-29} \mu_{B}$ is the Bohr magneton and $\hbar$ the reduced Planck 


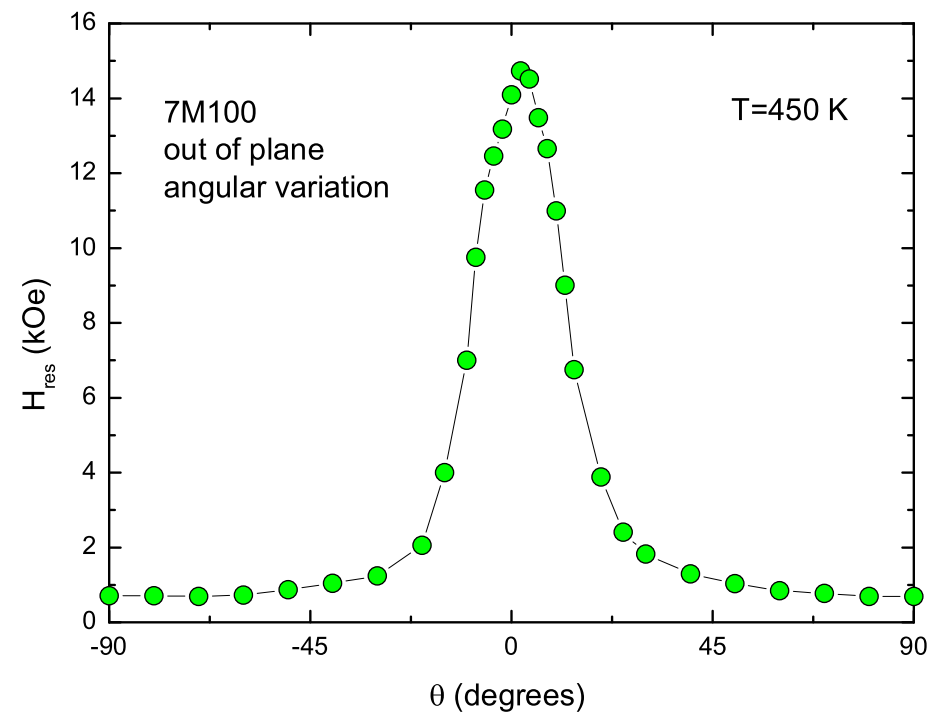

FIG. 7: Angular variation of the resonance field, $H_{\text {res }}$, in the OOP configuration for the sample $7 \mathrm{M} 100$ at $T=450 \mathrm{~K} . \theta$ is the angle between the magnetic field, $H$, and the film plane.

TABLE III: Saturation magnetization, perpendicular resonance field, effective anisotropy, perpendicular anisotropy and magnetoelastic anisotropy for FeRh films deposited on different substrates.

\begin{tabular}{|c|c|c|c|c|c|}
\hline Sample & $M_{\mathrm{S}}\left(\mathrm{emu} / \mathrm{cm}^{3}\right)$ & $H_{\perp}(\mathrm{Oe})$ & $H_{\text {eff }}(\mathrm{Oe})$ & $H_{A}^{\perp}(\mathrm{Oe})$ & $H_{A}^{\mathrm{ME}}(\mathrm{Oe})$ \\
\hline 7M100 & 925 & 14447 & 11087 & +537 & +720 \\
\hline 7M111 & 1004 & 16450 & 13090 & -473 & -226 \\
\hline 7A01 & 830 & 15600 & 12240 & -1810 & -793 \\
\hline A01 & 760 & 13580 & 10220 & -670 & - \\
\hline
\end{tabular}

constant. Because the magnetization for the parallel mode was not fully saturated in $\mathrm{X}$ band experiments, we only used the second Kittel equation and the values of $M_{s}$ reported in the previous section to estimate $H_{A}^{\perp}$ for the different samples. In some of the films we have found a small in-plane anisotropy which was not necessary to consider in the present analysis. Results are presented in Table III.

From the data shown in Table III it is readily observed that the perpendicular anisotropy $H_{\mathrm{A}}^{\perp}$ is maximum for the film $7 \mathrm{M} 100$, decreases and changes sign for $7 \mathrm{M} 111$ and reaches a 
minimum for 7A01. Note that a positive $H_{\mathrm{A}}^{\perp}$ represents an anisotropy favoring the alignment of $M$ perpendicular to the film plane, while a negative $H_{\mathrm{A}}^{\perp}$ tends to align $M$ inside the film. According to the crystallographic results, the sample 7M100 has an in-plane strain, 7M111 shows a relatively small tensile stress and 7A01 has a much larger tensile stress. In a simple model the magnetoelastic anisotropy coefficient may be written as $K_{M E}=-\frac{3}{2} \lambda \sigma_{x}=\frac{3}{2} \lambda \frac{\varepsilon_{z} Y}{2 \nu}$, where $Y$ is the Young's modulus and $\lambda$ the linear saturation magnetostriction. Reported values $^{17,30}$ of bulk FeRh are $Y \sim 1.9-2.1 \times 10^{12} \mathrm{dyn} / \mathrm{cm}^{2}$. For the magnetostriction in the FM phase there is a range of values ${ }^{18,30,31}$ from $\lambda \sim 0.5-1 \times 10^{-5}$ to $\lambda \sim 10^{-4}$, that depends on the temperature region and the maximum field used for the estimation of $\lambda$. If the additional anisotropy term is assumed to be of magnetoelastic origin it may be related to an anisotropy field by $H_{A}^{\mathrm{ME}}=2 K_{M E} / M_{s}$. Using an average value for $\lambda \sim 1 \times 10^{-5}$ and the strain calculated from the XRD data, we obtained the values shown in the last column of Table III. We observe a reasonably good agreement between $H_{\mathrm{A}}^{\perp}$ and $H_{A}^{\mathrm{ME}}$ which indicates that most of the observed effects are of magnetoelastic origin. The systematic larger values of $H_{A}^{\mathrm{ME}}$ may be due to an underestimation of the relaxed lattice parameter $a_{0}$.

As we already mentioned, we have found a relatively small in-plane uniaxial anisotropy in some of the films (for example 7M100) in which a variation of $H_{\text {res }}$ with cubic symmetry was expected. This observation is also consistent with the influence of magnetoelastic effects on the magnetic behavior of the films.

\section{CONCLUSIONS}

We have grown highly oriented FeRh thin films onto $\mathrm{MgO}$ and $\mathrm{Al}_{2} \mathrm{O}_{3}$ substrates of different crystalline orientations by conventional sputtering techniques. Most of the samples were annealed at $700{ }^{\circ} \mathrm{C}$ in order to induce the chemical order of the $\mathrm{Rh}$ and $\mathrm{Fe}$ atoms, the reduction of the residual $\gamma$ phase, and the promotion of the AF-FM transition. The properties of this unusual transition, which occurs between $325 \mathrm{~K}$ and $420 \mathrm{~K}$ depending on the sample and the magnetothermal history, were studied by magnetization and FMR experiments. We observed a thermal hysteresis consistent with a first order transition in the FMR measurements. We determined the transition temperature $T_{\mathrm{AF}-\mathrm{FM}}$ and found that it depends on the kind of substrate and growth orientation. This was ascribed to the differences in the stresses experienced by the films due to the specific mismatch between 
film/substrate lattice parameters. From the temperature dependence of the resonance field and the linewidth we have determined a phase coexistence region AF-FM, as suggested by the reduction of $H_{\text {res }}$ when the temperature is decreased. This fact coincides with the abrupt increase in the peak-to-peak linewidth, which is also consistent with this picture. The resonance field perpendicular to the film plane was used to estimate the perpendicular anisotropy in the different samples. Concerning the magnetic anisotropies of our FeRh films, the angular variation of the resonance field measured in the out-of-plane configuration is the one expected for a thin film dominated by the shape anisotropy, which tends to place the magnetization vector in the film plane, plus other extra contributions also perpendicular to this plane, of magnetoelastic origin.

Finally, the analysis of the angular dependence of $H_{\text {res }}$ measured using the in-plane configuration supports the hypothesis that there is a prevailing in-plane uniaxial anisotropy, arising also from stress effects, which dominates over the expected four-order symmetry expected from pure magnetocrystalline terms.

\section{Acknowledgments}

This work was partially supported by CONICET under Grant PIP 201501-00213, ANPCyT Grant PICT 2013-0401, and U.N. Cuyo Grant 06/C484 (Argentina). H. Kumar acknowledges FAPESP for providing the postdoctoral fellowship (process 2010/18590-0) to develop this work. The technical support from Rubén E. Benavides, César Pérez, Matías Guillén (Argentina) and Sergio Romero, Antonio Carlos Franco da Silveira, Társis Mendes Germano, and Tiago Fiorini da Silva (IFUSP, Brazil) is deeply acknowledged.

a Presently at Department of Applied Physics, Amity University UP, Sector 125, Noida-201301, India

b Electronic address: butera@cab.cnea.gov.ar; Also at INN - Instituto de Nanociencia y Nanotecnología

1 M. Fallot and R. Rocart, Rev. Sci 77, 498 (1939).

2 G. Shirane, C. W. Chen, P. A. Flinn, and R. Nathans, Phys. Rev. 131, 183 (1963)

3 L. J. Swartzendruber, Bulletin of Alloy Phase Diagrams, vol. 5, number 5, 456 (1984).

4 M. Fallot, Ann Phys. 10, 291 (1938). 
5 M. K. Chattopadhyay, S. B. Roy, A. K. Nigam, K. J. S. Sokhey, and P. Chaddah, Phys. Rev. B 68, 174404 (2003). S. B. Roy, G. K. Perkins, M. K. Chattopadhyay, A. K. Nigam, K. J. S. Sokhey, P. Chaddah, A. D. Caplin, and L. F. Cohen, Phys. Rev. Lett. 92, 147203 (2004).

6 J.-U. Thiele, S. Maat, and E. E. Fullerton, Appl. Phys. Lett. 82, 2859 (2003).

7 X. Marti, I. Fina, C. Frontera, Jian Liu, P. Wadley, Q. He, R. J. Paull, J. D. Clarkson, J. Kudrnovský, I. Turek, J. Kuneš, D. Yi, J-H. Chu, C. T. Nelson, L. You, E. Arenholz, S. Salahuddin, J. Fontcuberta, T. Jungwirth and R. Ramesh. Nature Materials 13, 367-374 (2014).

8 J. van Driel et al., J. Appl. Phys. 85, 1026 (1999).

9 S. Maat, J.-U. Thiele, and Eric E. Fullerton, Phys. Rev. B 72, 214432 (2005).

10 S. Inoue et al., J. Appl. Phys. 103, 07B312 (2008).

11 I. Suzuki, J. Appl. Phys. 105, 07E501 (2009).

12 E. Mancini et al., J. Phys D.: Appl. Phys. 46, 245302 (2013).

13 Y. Othani and I. Hatekayama, J. Appl. Phys. 74, 3328 (1993).

14 Hardeep Kumar, Márcia C. A. Fantini, and Daniel R. Cornejo, IEEE Trans. Mag., VOL. 49, 4506 (2013).

15 N. V. Baranov and E. A. Baranova, J. Alloys Comp. 219, 139 (1995).

16 C. W. Barton, T. A. Ostler, D. Huskisson, C. J. Kinane, S. J. Haigh, G. Hrkac and T. Thomson. Sci Rep. vol.7, 44397 (2017).

17 S. B. Palmer , P. Dentschuk, and D. Melville, Phys. Stat. Sol. (a) vol.32, 503 (1975)

18 M. R. Ibarra and P. A. Algarabel, Phys. Rev. B, vol. 504196 (1994)

19 David W. Cooke, F. Hellman, C. Baldasseroni, C. Bordel, S. Moyerman and E. E. Fullerton, Phys. Rev. Lett. 109, 255901 (2012)

20 K. Shikada, K. Tabuchi, M. Ohtake, F. Kirino, and M. Futamoto, J. Magn. Soc. Jpn., 32, 296-303 (2008)

21 S. Yuasa, T. Katayama, K. Murata, M. Usukura and Y. Suzuki, J.Magn. Magn. Mater. 177-181, $1296(1998)$

22 C. Bordel, J. Juraszek, David W. Cooke, C. Baldasseroni, S. Mankovsky, J. Minár, H. Ebert, S. Moyerman, E. E. Fullerton, and F. Hellman. Phys. Rev. Lett. 109, 117201 (2012).

23 Jiangwei Cao, Nguyen T. Nam, Sho Inoue, Hnin Yu Yu Ko, Nguyen N. Phuoc, and Takao Suzuki. J. Appl. Phys. 103, 07F501 (2008).

24 G. C. Han, J. J. Qiu, Q. J. Yap, P. Luo, T. Kanbe, T. Shige, D. E. Laughlin, and J.-G. Zhu. J. 
Appl. Phys. 113, 123909 (2013).

25 J. Smit, H.G. Beljers. Philips Res. Rep., 10, p. 113(1955)

26 A. Butera, J. L. Weston, and J. A. Barnard, J. Magn. Magn. Mat vol 284, pp. 17-25 (2004).

27 A. Butera, European Phys. J. B 52 p. 297-303 (2006).

28 N. Álvarez, G. Alejandro, J. Gómez, E. Goovaerts, and A. Butera. J. Phys D.: Appl. Phys. 46, 505001 (2013).

29 A. Butera, N. Alvarez, G. Jorge, M. M. Ruiz, J. L. Mietta, R. M. Negri, Phys. Rev. B 86, $144424(2012)$.

30 A. I. Zakharov, A.M. Kadomtseva, R. Z. Levitin, and E. G. Ponyatovskii, Soviet Physics JETP, Volume 19, Number 6, p.1348 (1964).

31 C. Marquina, M. R. Ibarra, P. A. Algarabel, A. Hernando, P. Crespo, P. Agudo, A. R. Yavari, and E. Navarro. J. Appl. Phys. 81, 2315 (1997). 